\title{
A computer aided diagnostic tool for Evaluation of Rheumatoid Arthritis in Hand Thermal images
}

Snekhalatha.umapathy ${ }^{1}$, Anburajan.Micheal ${ }^{2}$ Sowmiya . ${ }^{3}$ Venkatraman.B $^{4}$, Menaka .Murugesan 5,

${ }^{1,2,3}$ Department of Biomedical Engineering, SRM University, Kattankulathur, chennai-603203 ,Tamilnadu, India

${ }^{4,5}$ Indra Gandhi Center for Atomic Research, Kalpakkam-603102, Tamil Nadu,India

E-mail: ${ }^{1}$ sneha_samuma@yahoo.co.in, ${ }^{2}$ hod.biomedi@ktr.srmuniv.ac.in,sowmiya.vasu@gmail.com,

3bvenkat@igcar.gov.in, ${ }^{4}$ menaka@igcar.gov.in

\begin{abstract}
Objectives:

The aim of the study was to develop a computer aided diagnostic tool for evaluation of Rheumatoid arthritis in hand thermal images.

\section{Methods:}

The skin surface temperature measurement and heat distribution index was analyzed in whole hand thermal image of rheumatoid arthritis patients (RA) and controls. K means algorithm was compared with EM algorithm in evaluation of RA in hand thermal images. Finally a computer aided diagnostic tool was made using Matlab for diagnosing the rheumatoid arthritis.
\end{abstract}




\section{Results:}

In evaluation of skin surface temperature measurements, $3^{\text {rd }}$ and $4^{\text {th }}$ Metacarpophalangeal joints , $3^{\text {rd }}$ Proximal Interphalangeal joints and $3^{\text {rd }}$ Distal Interphalangeal joints shows highly significant difference in temperature than other joints between RA patients and healthy controls $(\mathrm{p}<0.01)$. K means algorithm provided better segmentation results compared to EM algorithm in evaluating the disease.

\section{Conclusion:}

The developed computer aided diagnostic tool provided better prediction of rheumatoid arthritis with key interpretation in hand thermal images in the total population studied.

\section{KEYWORDS:}

Skin surface temperature measurements, heat distribution index, rheumatoid arthritis, k means algorithm, Expectation Maximization algorithm 